\title{
Dust in comet McNaught-Hartley (C/1999 T1) from Jan. 25 to Feb. 04, 2001: IR and optical CCD imaging
}

\author{
L.-M. Lara ${ }^{1}$, J. Licandro ${ }^{2}$, and G. P. Tozzi ${ }^{3}$ \\ 1 Instituto de Astrofísica de Andalucía, CSIC, Camino Bajo de Huétor 24, 18008 Granada, Spain \\ 2 Isaac Newton Group of Telescopes \& Instituto de Astrofísica de Canarias, PO Box 321, 38700 Santa Cruz de La Palma, \\ Tenerife, Spain \\ e-mail: licandro@ing.iac.es \\ 3 INAF-Osservatorio Astrofisico di Arcetri, Largo E. Fermi 5, 50125, Firenze, Italy \\ e-mail: tozzi@arcetri.astro.it
}

Received 22 July 2002 / Accepted 13 March 2003

\begin{abstract}
Broad-band optical and infrared observations in the $R, I$, and $J, H, K_{\mathrm{s}}$ bands of comet McNaught-Hartley (C/1999 T1) were performed from Jan. 25 to Feb. 4, 2001, shortly after the comet perihelion on Dec. 14, 2000. For the time the comet was observed, it did not show any peculiar behaviour either in the infrared or in the optical, that is, no non-spherical structures, besides the dust tail, or outburst or daily activity variations were detected. Calibration of the images in the $A f \rho$ frame provide us with values of dust production between 1000 and $3000 \mathrm{~cm}$, depending on the date and on the wavelength. The general behaviour of the dust coma of C/1999 T1 is that described by a steady-state production of long-lived grains expanding radially outwards from the nucleus. The dust color does not clearly change with projected cometocentric distance. During a time span of 10 days, the cometary dust grains observed in the $K_{\mathrm{s}}$ filter seemed to become slightly redder on Feb. 05 than they were on Jan. 26, 2001.
\end{abstract}

Key words. comets: individual: McNaught-Hartley (C/1999 T1) - comets: general

\section{Introduction}

Comet McNaught-Hartley was discovered by McNaught on Oct. 7.64, 1999, from a plate taken by Hartley with the $1.2 \mathrm{~m}$ UK Schmidt Telescope at the Siding Spring Observatory. At the time of discovery, the comet showed a coma of 8 " and a very faint tail of $\sim 1^{\prime}$. The orbital elements derived from subsequent observations indicate that comet McNaughtHartley (C/1999 T1) is a long period (7045 years) comet in a parabolic orbit with perihelion distance at $r_{\mathrm{h}}=1.1717 \mathrm{AU}$ on Dec. 14, 2000, and closest approach to Earth, $\Delta=1.2868 \mathrm{AU}$ on Feb. 2, 2001. Therefore, C/1999 T1 is a member of the Oort-cloud comets.

After discovery, the comet was observed mainly in the $\mathrm{mm}$ and IR range; optical observations were only reported by Schleicher et al. (2001, IAUC 7558). From eight sets of narrowband optical photometry on Dec. 28, 2000 and on Jan. 2, 2001, they derived $\mathrm{a}_{2} \mathrm{O}$ and $\mathrm{CN}$ production rate of $5.75 \times 10^{28}$ and $1.26 \times 10^{26} \mathrm{~s}^{-1}$, respectively, whereas $A f \rho$ was reported to be $1150 \mathrm{~cm}$. Almost simultaneously (Jan. 5-7, 2001), Biver et al. (2001, IAUC 7559) reported the detection of the CO J(3-2), $\mathrm{HCN} \mathrm{J}(3-2)$ and $\mathrm{CH}_{3} \mathrm{OH}$ lines with the Caltech Submillimiter Observatory (CSO). The average production rates relative to water are: $\mathrm{CO} 40 \%, \mathrm{CH}_{3} \mathrm{OH} 5 \%$ and $\mathrm{HCN} 11 \%$. According to these results, the $\mathrm{CO}$ ratio is the highest ever observed in a comet that is relatively close to the Sun. Other species were detected by Mumma et al. (2001, IAUC 7578) in the IR; using emission lines near $4.67 \mu \mathrm{m}$, water and carbon dioxide production were $8.2 \times 10^{28}$ and $1.4 \times 10^{28} \mathrm{~s}^{-1}$ on Jan. 13 , respectively. The water production rate derived from $\mathrm{OH}$ measurements on Jan. 14, using the NIRSPEC at the W. M. Keck Observatory by Conrad \& Chaffee (2001, IAUC 7578) is twice higher than that obtained by Mumma et al. (2001, IAUC 7578) one day before, still within uncertainties derived from the use of the Haser or Vectorial modeling. Ethane and methanol were also observed and their production rates obtained. Methane was tentatively detected as well, and several $\mathrm{HCN}$ and $\mathrm{C}_{2} \mathrm{H}_{2}$ lines were clearly seen. From these measurement, the gas composition in comet McNaught-Hartley, in terms of CO, seems to be similar to C/1996 B2 Hyakutake and C/1995 O1 Hale-Bopp (DiSanti et al. 2001, DPS 33. \#20.11), but this carbon monoxide content is much higher than those found for three other Oort-cloud comets C/1999 H1 Lee, C/1999 S4 LINEAR and C/1999 A2 LINEAR. Comparing the ethane and ethanol abundances to those in other comets, $\mathrm{C} / 1999 \mathrm{~T} 1$ can be considered similar to C/1996 B2, C/1995 O1 and C/1999 H1. 
The cometary spectrum at $\lambda \approx 10 \mu \mathrm{m}$ was investigated by Lynch et al. (2001, IAUC 7582; 2002) on Jan. 31, identifying two prominent emissions features at 10.3 and $11.2 \mu \mathrm{m}$, the latter being indicative of crystalline olivine. The $10.3 \mu \mathrm{m}$ was only 1-2 sigma detection, but if real could indicate the presence of hydrated silicates. The dust temperature was $(260 \pm 10) \mathrm{K}$, only about $10 \%$ above the blackbody radiative equilibrium. These two prominent emission (likely due to crystalline olivine and hydrated silicates) disappeared the day after, although the silicate emission features remained in the spectrum. Three weeks after these observations by Lynch et al. (2001, IAUC 7582; 2002), Woodward et al. (2001, IAUC 7594) took spectra of the comet between 7 and $23 \mu \mathrm{m}$ and they found no evidence for strong silicate emission, while the blackbody fit to the observed spectral energy distribution seemed not to have varied $(270 \pm 20 \mathrm{~K})$. From all of the reported observations of C/1999 T1, it can be concluded that the comet gas composition, excluding $\mathrm{CO}$, is rather similar to that prevailing in other Oort-cloud comets. However, not much seems to be known about its dust, such as 2D spatial distribution, color behaviour and maps, production rate, etc.

The imaging of comets in the infrared can be a powerful method of studying spatial variations in the optical properties of the dust. The population of dust particles of radius less than $1 \mu \mathrm{m}$ is most sensitive to the scattered radiation at $\lambda<3 \mu \mathrm{m}$; whereas the thermal emission (typically longward of $3 \mu \mathrm{m}$ ) is most sensitive to particles of radius greater than $1 \mu \mathrm{m}$. In this paper, we analyse near-infrared and optical images of McNaught-Hartley (C/1999 T1) in order to get information about the spatial distribution within the inner coma and tails of dust grains with various physical properties (Hanner \& Tokunaga 1991; Campins et al. 1989). The imaging observations and their data reduction are presented in Sect. 2, Sect. 3 contains the analysis of the results and the discussion of their physical implications. Conclusions are presented in Sect. 4.

\section{Observations and data reduction}

Comet McNaught-Hartley (C/1999 T1) was observed in the near-infrared and in the optical range. The infrared and optical observations were made by using three different telescopes: the $3.56 \mathrm{~m}$ Telescopio Nazionale Galileo (TNG) the Italian telescope at the "El Roque de los Muchachos" Observatory (La Palma, Canary Islands, Spain); the $1.5 \mathrm{~m}$ Telescopio Infrarosso del Gornergrat (TIRGO), the Italian infrared facility located on the Swiss alps at $3200 \mathrm{~m}$ of altitude; and the $82 \mathrm{~cm}$ IAC-80 telescope of IAC at Teide Observatory (Tenerife, Canary Islands, Spain). From Jan. 26 to 31, we did nearinfrared imaging observations with the TIRGO using ARNICA (ARcetri Near Infrared CAmera) imaging camera covering the near infrared bands between 1.0 and $2.5 \mu \mathrm{m}$. The scale is $1^{\prime \prime}$ per pixel, with sky coverage of more than $4^{\prime} \times 4^{\prime}$ on the detector array, a NICMOS 3 type $(256 \times 256$ pixels, $40 \mu \mathrm{m}$ side). Broad-band $J(1.26 \mu \mathrm{m}), H(1.65 \mu \mathrm{m})$ and $K_{\mathrm{s}}(2.205 \mu \mathrm{m})$ images were obtained every night from Jan. 26 to 31, 2001, but not on Jan. 29 due to bad weather conditions. Near-infrared images in the same bands were also done on Feb. 4 and 5 with the TNG using NICS (the Near Infrared Camera Spectrograph).
The detector is a HgCdTe Hawaii $1024 \times 1024$ array that, using the large field camera, has a plate scale of $0.25^{\prime \prime}$ per pixel, covering a $4^{\prime} \times 4^{\prime}$ field.

The IR observations were complemented with observations in the optical range (broad-band $R$ and $I$ filters) on Jan. 26 and Feb. 5. On Jan. 26, the images were taken with the TNG using the camera-spectrograph DOLORES (Device Optimized for the LOw REsolution) and with the IAC-80 telescope using the Thomson $1 \mathrm{k} \times 1 \mathrm{k}$ direct CCD camera. On Feb. 5 the $R$ and $I$ images were taken with the IAC-80 telescope. In the imaging mode, DOLORES provides a $9.4^{\prime} \times 9.4^{\prime}$ field $\left(0.275^{\prime \prime}\right.$ per pixel plate scale), while the Thomson CCD at the IAC- 80 provides a $7.3^{\prime} \times 7.3^{\prime}$ field $\left(0.43^{\prime \prime}\right.$ per pixel plate scale). $R$ and $I$ filters allow little contamination from cometary molecular emissions in the coma and are, therefore, appropriate for studying the dust.

The near infrared images were obtained in the usual way (i.e. chopping between the comet and the sky). In the case of TIRGO observations, for all of the filters, four consecutive or alternate series of 8 images of 15 or $10 \mathrm{~s}$ exposure time in the $J$ band and $5 \mathrm{~s}$ integration time in the $H$ and $K_{\mathrm{s}}$ bands were obtained during our run. Sky images were also obtained in the same way in all but the tail directions, at a distance of 8 arcmin from the comet. In the case of TNG observations, one series of 7 images of the comet of $10 \mathrm{~s}$ exposure time in the $J$ and $H$ bands and one of $20 \mathrm{~s}$ exposure time in the $H$ band were taken on Feb. 4 and one series of 7 images of $10 \mathrm{~s}$ exposure time in the $J, H$ and $K_{\mathrm{s}}$ band on Feb. 5. $J, H$ and $K_{\mathrm{s}}$ sky images were median averaged to create the sky and flat field images used to correct the corresponding comet images. Details of the observations are presented in Table 1. All of the observations were done while tracking on the comet proper motion. All nights but Jan. 27 and 28 were photometric. Several infrared standard stars (Hunt et al. 1998) were observed each night for calibration purposes and in order to better determine the calibration uncertainties. The calibration error was $\sim 5 \%$.

The reduced frames obtained during photometric nights were calibrated in $A f$, i.e. the average albedo $(A(\theta)$, no correction for the phase angle was applied) multiplied by the filling factor $(f)$ that represents the percentage of area covered by the dust (see Tozzi \& Licandro 2002). This notation Af $\rho$ has been introduced by A'Hearn et al. (1984) and it defines a measurement unit related to the dust production rate of a comet, independent of the aperture used to measure the flux, and it is the average albedo multiplied by the filling factor and the projected nucleocentric distance $(\rho)$. It can be interpreted as the average of the percentage of solar radiation scattered by the cometary dust toward the observer. In terms of measurable quantities, it is equal to

$A f=\left(\frac{2 \Delta r_{\mathrm{H}}}{\rho}\right)^{2} \frac{F_{\mathrm{c}}}{F_{\mathrm{S}}}$

where $\Delta$ and $r_{\mathrm{H}}$ are the comet's geocentric and heliocentric distances, respectively, $\rho$ is the projected radius of aperture, $F_{\mathrm{c}}$ is the measured cometary flux in every filter, and $F_{\mathrm{S}}$ is the solar flux in the same filter.

To obtain the final images of the comet, the position of the comet optocenter in the calibrated images was determined by fitting a two-dimensional Gaussian to the innermost 
Table 1. Details of the observations.

\begin{tabular}{ccccccccc}
\hline \hline $\begin{array}{c}\text { Date } \\
(2001)\end{array}$ & $\begin{array}{c}\text { Time } \\
\mathrm{UT}\end{array}$ & Band & $\begin{array}{c}\Delta \\
\mathrm{AU}\end{array}$ & $\begin{array}{c}r_{\mathrm{H}} \\
\mathrm{AU}\end{array}$ & $\begin{array}{c}\mathrm{km} \\
\text { per pixel }\end{array}$ & $\begin{array}{c}\text { PA } \\
\text { deg }\end{array}$ & $\begin{array}{c}\text { Phase } \\
\mathrm{deg}\end{array}$ & $\mathrm{Qual}^{a}$ \\
\hline Jan. 26 & $03: 57-05: 01$ & $J, H, K_{\mathrm{s}}$ & 1.298 & 1.355 & 945 & 293.2 & 43.5 & $\mathrm{a}$ \\
& $06: 34-07: 17$ & $R, I$ & & & $408^{1}, 260^{2}$ & & & $\mathrm{a}$ \\
Jan. 28 & $03: 32-04: 40$ & $J, H, K_{\mathrm{s}}$ & 1.293 & 1.370 & 942 & 293.1 & 43.3 & $\mathrm{~b}$ a \\
Jan. 30 & $03: 20-03: 49$ & $J, K_{\mathrm{s}}$ & 1.290 & 1.387 & 940 & 292.9 & 43.0 & $\mathrm{a} \mathrm{b}$ \\
Jan. 31 & $03: 20-04: 19$ & $J, H, K_{\mathrm{s}}$ & 1.288 & 1.395 & 938 & 292.8 & 42.8 & $\mathrm{a}$ \\
Feb. 04 & $06: 46-07: 01$ & $J, K_{\mathrm{s}}$ & 1.287 & 1.430 & $234^{3}$ & 292.1 & 42.1 & $\mathrm{a}$ \\
Feb. 05 & $05: 37-05: 55$ & $J, H, K_{\mathrm{s}}$ & 1.288 & 1.438 & $234^{3}$ & 291.9 & 41.9 & $\mathrm{a}$ \\
& $05: 24-06: 18$ & $R, I$ & & & $406^{1}, 258^{2}$ & & & $\mathrm{a}$ \\
\hline
\end{tabular}

PA refers to the position angle of the extended Sun-target radius vector. Phase is the Sun-comet-observer angle. $r_{\mathrm{H}}$ and $\Delta$ are the heliocentric and geocentric distances of the comet during our observations.

${ }^{a}$ Night quality code: $\mathrm{a}$ - good, $\mathrm{b}$ - cirrus. $\mathrm{a} \mathrm{b}$ (or $\mathrm{b}$ a) means good sky conditions at the beginning of the night and getting worse (cirrus) at the end of the night (or vice versa).

1 Pixel scale $0.4325^{\prime \prime}$ per pixel for IAC-80.

2 Pixel scale $0.275^{\prime \prime}$ per pixel for TNG-LRS.

3 Pixel scale $0.25^{\prime \prime}$ per pixel for TNG-NICS.

20 pixels of the coma with an uncertainty $\leq 0.3$ pixels. Before coadding, all images were recentered using the derived optocenters. Those images taken in two consecutive series with the same filter were median combined and analysed.

\section{Analysis and discussion}

The morphological study of the dust coma was done on fully calibrated images.

We searched for structures (jets, fans...) in the coma by applying a Laplacian filtering (Boehnhardt \& Birkle 1994) on at least two images for each night. No clear non-spherical structures were found. An example of both raw and filtered $I$ band images are presented in Fig. 1.

No clear day-to-day morphological variations are seen either in large or in small scale images. In the small scale, i.e. in the inner 50 " (corresponding to $\approx 47000 \mathrm{~km}$ ), all images are fairly symmetrical about the nucleus. At larger spatial scale $\left(>10^{5} \mathrm{~km}\right)$, the effect of radiation pressure is visible leading to an incipient dust tail at a position angle of $\sim 290^{\circ}$ east of north. To better assess if the dust coma shows changes in its morphology, Fig. 2 displays the isocontours of $A f$ between 0 and $2 \times 10^{-6}$ in $3 \times 10^{-7}$ intervals as obtained from calibrated $K_{\mathrm{s}}$ band images throughout the observing run.

The radial and azimuthal infrared surface brightness variations derived from our images can be used to test models of dust grain production from cometary nuclei. If the rate of grain production from the nucleus is constant (i.e. steady-state) and spherically symmetric and if the coma optical depth is everywhere much less than unity, then standard models (Gehrz \& Ney 1992) predict that the surface brightness $B(\rho)$ or cometary flux $F_{\mathrm{c}}(\rho)$ will decline as $\rho^{-1}, \rho$ being the projected cometocentric distance. When solar radiation pressure becomes important, the azimuthally-averaged cometary flux profile varies as $\rho^{-1.5}$ (Jewitt 1991). In order to describe the shapes of the $F_{\mathrm{c}}$ profiles we define the logarithmic derivative $m=\mathrm{d} \log \left(F_{\mathrm{c}}\right) / \mathrm{d} \log \rho$. For a steady-state, isotropic outflow, $m=-1$, while the radiation pressure dominated case gives $m=-1.5$. Integrating the $B(\rho)$ values in an area enclosed by two concentric circles of radius $\rho$ and $\rho+\mathrm{d} \rho$, over a circular aperture of angular radius $\rho$, we obtain the azimuthally integrated brightness $B^{\prime}(\rho)$, with logarithmic slope $m^{\prime}=\mathrm{d} B^{\prime} / \mathrm{d} \log \rho=m+2$. For a comet with a production of dust described by long-lived grains expanding radially outwards $m^{\prime}=1$ and $m^{\prime}=0.5$ for the radiation-dominated case. Comet McNaught-Hartley followed the steady-state behaviour for every date it was imaged, with $m^{\prime}=1.0 \pm 0.01$ (2-sigma).

To also quantify the dust activity, the images were analysed by measuring the $A f \rho$ and the gradient of the integral of the $A f\left(\sum A f\right)$ as measured in a circular aperture, as function of the projected cometocentric distance, $\rho$. According to the previous result, i.e. $m^{\prime} \sim 1.0, \sum A f$ and $A f \rho(\rho)$ theoretically should be constant. At relatively small spatial scales $\left(\approx 10^{4} \mathrm{~km}\right)$, the solar radiation pressure does not strongly affect the profile of $A f \rho$. However, the $A f \rho$ and $\sum A f$ profiles are affected in the inner coma by the seeing, which makes the quantities approach zero close to the photometric nucleus. Similarly, in the outer coma they can be affected by an incorrect sky subtraction making $A f \rho$ and $\sum A f$ linearly dependent on $\rho$. Figure 3 shows the $\sum A f$ and $A f \rho$ as a function of $\rho$ in the various bands as measured on Jan. 26. The values of $\sum A f$ are all constant with $\rho$. That means that the coma is represented by the simple outflow model (i.e. neither vaporization of ice particles nor dust fragmentation) and no short time scale variation of dust production rate has taken place.

The dust ejection velocity from the inner coma is parameterized by $v(t, d)=v\left(t, d_{0}\right) \times\left(\frac{d}{d_{0}}\right)^{u}$, where $t$ is the time of dust ejection from the inner coma, $d$ is the dust diameter, $d_{0}$ is the dust reference diameter, $v\left(t, d_{0}\right)$ describes the time evolution of the dust ejection, and $u$ describes the size dependence (a power law) of the dust velocity (see Fulle et al. 1998). Dust dynamics depend on the ratio between solar radiation 


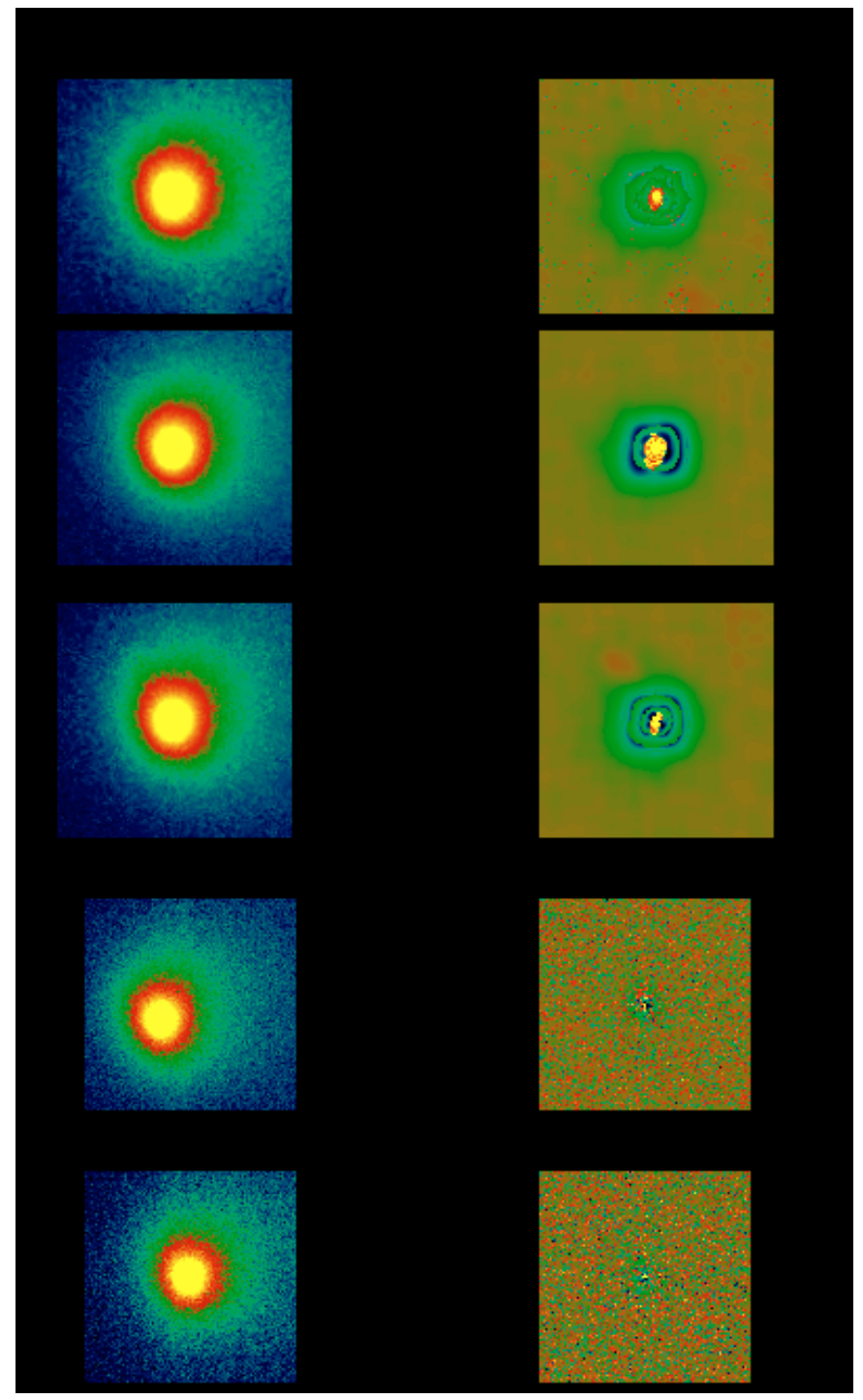

Fig. 1. Images taken from Jan. 26 to Feb. 05 in the $K_{\mathrm{s}}$ filter (left column) together with the processed ones to enhance any high frequency spatial structure (right column). Note that no non-spherical structure can be clearly detected on these dates. North is up and east is to the left. The Sun is at a position angle of $\sim 113^{\circ}$.

pressure and gravity forces, $(1-\mu)=C Q\left(\rho_{\mathrm{m}} d\right)^{-1}$, where $C=1.19 \times 10^{-3} \mathrm{~kg} \mathrm{~m}^{-2}$ is independent of the dust chemistry and physics, (Burns et al. 1979). By adopting $Q=1$ (large absorbing grains) and $\rho_{\mathrm{m}}=10^{3} \mathrm{~kg} \mathrm{~m}^{-3},(1-\mu)$ is converted to sizes and it is an adopted value. Thus, the flow velocity is estimated by making use of the expression (Fulle et al. 1998)

$v(t, 1-\mu)=v\left(t, 1-\mu_{0}\right)\left[\left(1-\mu_{0}\right) /(1-\mu)\right]^{u}$.

From the dust tail modeling by Moreno et al. $(2002,2003)$ and for a reference size particle of $590 \mu \mathrm{m}$ ejected about 45 days after perihelion (i.e. Jan. 30, 2001), $v(t, 1-\mu)$ is about $0.04 \mathrm{~km} \mathrm{~s}^{-1}$ if $u=-1 / 6$ (best fit parameter). Applying Eq. (2), $v\left(t, 1-\mu_{0}\right)\left[\left(1-\mu_{0}\right)\right]^{u}$ can be deduced for our reference size particle (i.e. $590 \mu \mathrm{m}$ ) and ejection time (i.e. Jan. 30, 2001) and it is equal to $0.113 \mathrm{~km} \mathrm{~s}^{-1}$. Therefore, for cometary grains with radii between 0.5 and $10 \mu \mathrm{m}$ and for the same reference date,
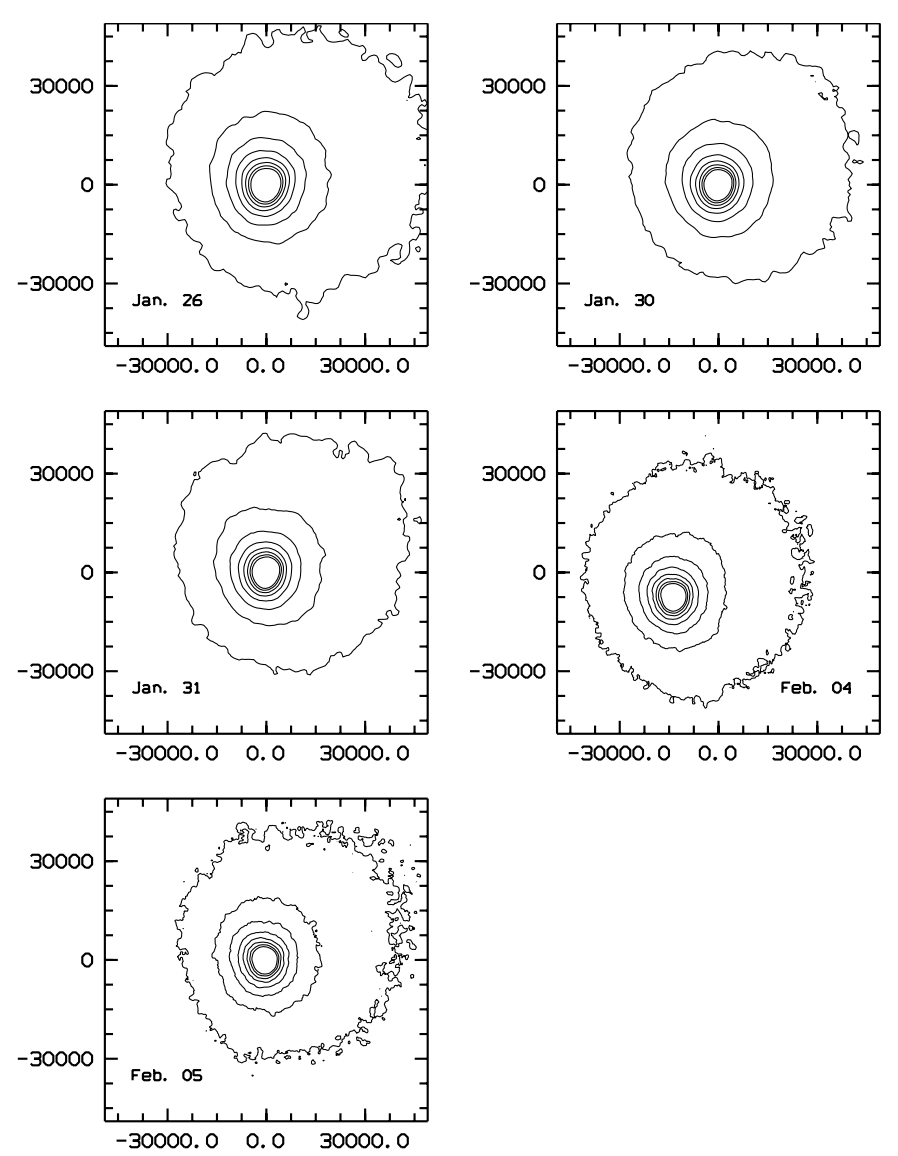

Fig. 2. Contours obtained from the median combined images taken in the $K_{\mathrm{s}}$ band on every night and calibrated in the $A f$ frame. The contours range from 0 to $2 \times 10^{-6}$ in steps of $3 \times 10^{-7}$. The nucleus is centered at $(0,0)$ and the spatial scale is $38000 \times 38000 \mathrm{~km}$ at the comet's distance.
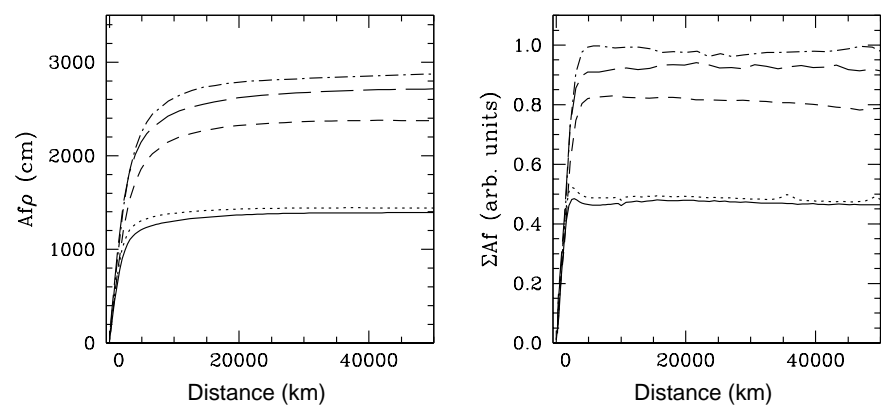

Fig. 3. Left panel: measured values of $A f \rho$ as a function of projected cometocentric distance $\rho$. Solid line refers to $R$, dotted line refers to $I$, dashed line to $J$, long-dashed line to $H$ and dot-dashed line to $K_{\mathrm{s}}$. The panel at the right refers to $\sum A f$, as function of $\rho$, in arbitrary units. The code for the lines is the same as for the panel to the left. Both Af $\rho$ and $\sum A f$ vs. $\rho$ indicate that the cometary dust of $\mathrm{C} / 1999 \mathrm{~T} 1$ can be described by a steady-state production rate model of long-lived grains.

the outflow velocity ranges from 0.115 to $0.07 \mathrm{~km} \mathrm{~s}^{-1}$, consequently the dust coma at $2 \times 10^{4} \mathrm{~km}$ still contains signatures of the dust production rate $(1.74-2.8) \times 10^{5} \mathrm{~s}(\approx 2-3.5$ days $)$ before. So, the constancy of $\sum A f$ means that, with a timescale of 2 to 3.5 days, neither outburst nor strong variations of activity were present in this comet. 


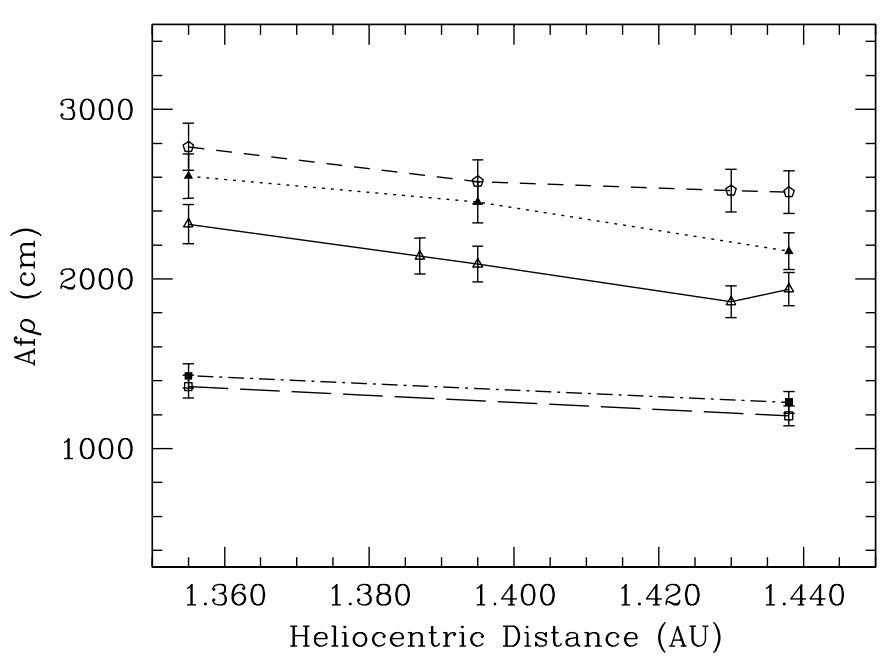

Fig. 4. Af $\rho$ measured within an aperture of $\rho \sim 20000 \mathrm{~km}$ in the different bands for the coma of C/1999 T1. $R$ - long-dashed line, $I$ - dot-dashed line, $J$ - solid line, $H$ - dotted line and $K_{\mathrm{s}}$ - dashed line. Note that $A f \rho$ in the $R, I$ and $K_{\mathrm{s}}$ bands are most invariant with $r_{\mathrm{H}}$.

Table 2. Af $\rho$ in the $J, H$, and $K_{\mathrm{s}}$ filters at $\rho \approx 20000$.

\begin{tabular}{lccccc}
\hline \hline Date & \multicolumn{5}{c}{$\rho \sim 20000 \mathrm{~km}$} \\
\hline 2001 & $R$ & $I$ & $J$ & $H$ & $K_{\mathrm{s}}$ \\
\hline Jan. 26 & 1370 & 1435 & 2330 & 2600 & 2790 \\
Jan. 30 & & & 2134 & & 2530 \\
Jan. 31 & & & 2075 & 2463 & 2587 \\
Feb. 04 & & & 1870 & & 2530 \\
Feb. 05 & 1190 & 1273 & 1942 & 2163 & 2510 \\
\hline
\end{tabular}

The relative "quietness" of this comet can be also seen in Fig. 4, where the $A f \rho$, measured in the different bands within an aperture of $\rho=20000 \mathrm{~km}$, are reported as a function of the heliocentric distance. A power law of $A f \rho$ vs. $r_{\mathrm{H}}\left(A f \rho \sim r_{\mathrm{H}}^{-a}\right)$ can be obtained from the data in $J, H$ and $K_{\mathrm{s}}$ bands as they represent the most complete data set (the comet was observed in $R$ and $I$ only on Jan. 26 and Feb. 05, 2001). For each band, the result of the fit is: $a_{J}=3.43 \pm 0.50, a_{H}=3.10 \pm 0.69$ and $a_{K}=1.51 \pm 0.56$. Although our obsevations only cover a small range of increasing heliocentric distance ( $0.08 \mathrm{AU})$, a steady decrease of the dust production rate with $r_{\mathrm{H}}$ is visible, confirmed by a relatively large value of $n_{J}$ and $n_{H}$, excluding the Af $\rho$ measured in the $K_{\mathrm{s}}$ bands which seems to decrease more slowly (also manifested as a less steep power law, i.e. lower $n_{K}$ ). This may be due to a change in the dust grain size distribution, giving rise to an increased abundance of larger grains.

We also searched for structures in the color maps obtained by dividing the calibrated images in different filters $I / R, H / J$, $K_{\mathrm{s}} / J$ and $K_{\mathrm{s}} / H$. Neither clearly defined structures nor color gradients with $\rho$ were found for any of the nights of observation, indicating very slow, if any, changes in the parameters characterising the dust grain size distribution at different projected cometocentric distances.

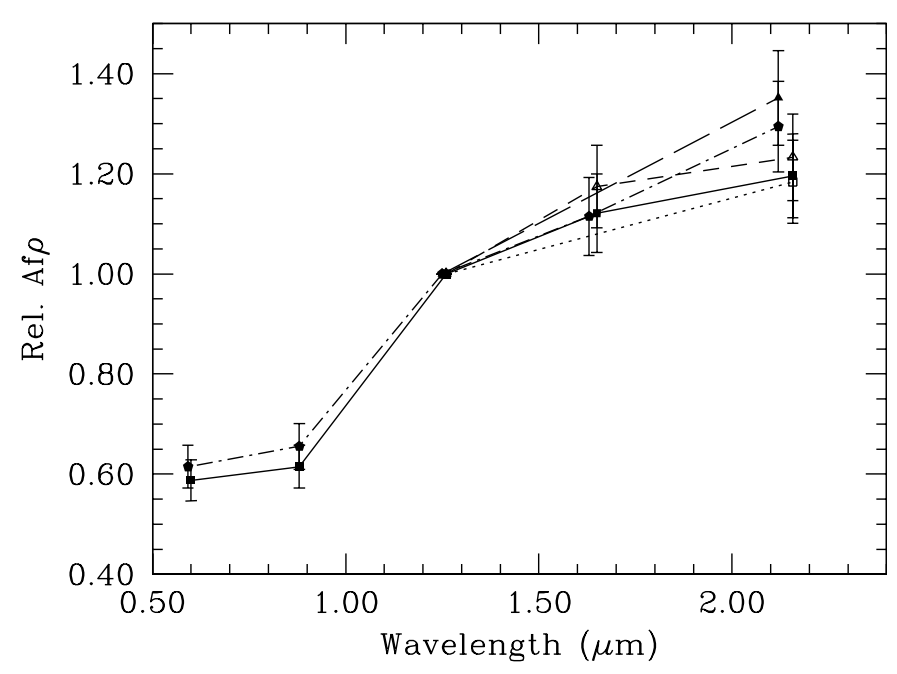

Fig. 5. Color of the dust in the coma of comet McNaught-Hartley at $\sim 20000 \mathrm{~km}$, as a function of the central wavelength of the filters. Points referred to the same night are connected by lines as follows: Jan. 26 - solid line, Jan. 30 - dotted line, Jan. 31 - dashed line, Feb. 04 - long-dashed line and Feb. 05 - dot-dashed line. All the values are normalised to that in the $J$ filter at $1.25 \mu \mathrm{m}$. Error bars take into account the $5 \%$ uncertainty in the calibration process.

We have also made cuts along the Sun-tail direction in every band and the flux ratios among them do no show any obvious color variation with the projected cometocentric distance $\rho$. Mean values were obtained in a circular aperture of radius $\rho \approx 20000 \mathrm{~km}$ and they are listed in Table 3 as the quotient of $A f \rho$ in the two mentioned bands in every column and beneath, as the classical definition of color index (i.e. difference of magnitudes) together with the solar color in the first row. The error in the calibration process is propagated here and these mean dust colors can be affected by an uncertainty $\leq 10 \%$. In terms of color indexes, the cometary dust is redder than the solar color on every date. Day to day color behaviour indicates whether some variations in the dust scattering properties took place during our observing run. Figure 5 shows the $A f \rho$ in every band relative to that in the $J$ band at $\rho \sim 20000 \mathrm{~km}$. The size of the dust grains seems to be larger on Feb. 05 as deduced from a redder color in the four color indexes, assuming that the dust composition did not dramatically change from Jan. 26 to Feb. 05.

This behaviour is not exclusive for comet McNaughtHartley; other comets have also shown it (e.g. P/Tempel 2, see Tokunaga et al. 1988); and the $J-H$ and $H-K$ colors do not necessarily correlate.These and other authors' results (Tokunaga et al. 1988) show that intrinsic color differences occur in the dust within one comet. Whether this is a result of a change in the ice/dust ratio, heterogeneity in the nucleus composition, or both cannot be ascertained without the inclusion of other observations, like mid-IR, spectroscopy and light curves, spectral mapping of the nucleus surface (e.g. 19P/Borrelly, Soderbloom et al. 2002), etc. From the colors alone, it is very difficult to determine whether these color changes arise from a change in composition or from a change in the average size of the particles. Effects of particle composition and size on the color have been discussed by Hartmann \& Cruikshank (1984) 
Table 3. Mean dust color at $\rho \sim 20000 \mathrm{~km}$.

\begin{tabular}{lcccc}
\hline \hline Sun & 0.34 & 0.33 & 0.37 & 0.04 \\
\hline Date & \multicolumn{4}{c}{$\rho \sim 20000 \mathrm{~km}$} \\
\hline 2001 & $I / R$ & $H / J$ & $K_{\mathrm{s}} / J$ & $K_{\mathrm{s}} / H$ \\
\hline Jan. 26 & 1.04 & 1.11 & 1.19 & 1.06 \\
& 0.37 & 0.44 & 0.56 & 0.11 \\
Jan. 30 & & & 1.18 & \\
& & & 0.55 & \\
Jan. 31 & & 1.17 & 1.23 & 1.05 \\
& & 0.50 & 0.59 & 0.09 \\
Feb. 04 & & & 1.35 & \\
& & & 0.70 & \\
Feb. 05 & 1.06 & 1.11 & 1.28 & 1.16 \\
& 0.40 & 0.44 & 0.64 & 0.20 \\
\hline
\end{tabular}

For a given date, the first and second row contain the true color and the color index of the cometary dust, respectively.

and Jewitt \& Meech (1986) concluding that these variations, uncorrelated to heliocentric distance or phase angle or gas coma composition or dynamical age, in most cases, reflect that the grain populations in different comets are intrinsically different.

Although very tentatively, the $A f \rho$ ratios in the $R, I, H$ and $K_{\mathrm{S}}$ bands relative to the $J$ band (see Table 3 and Fig. 5) can be used to interpret the dust composition. By making use of the information in Fig. 5, and applying simple Mie-scattering theory to a log-normal size distribution (see Hansen \& Travis 1974)

$n(a)=\frac{1}{(2 \pi)^{1 / 2} \sigma_{\mathrm{m}}} \frac{1}{a} \exp \left[-\left(\ln a-\ln a_{\mathrm{m}}\right)^{2} /\left(2 \sigma_{\mathrm{m}}^{2}\right)\right]$

we have attempted to get some approximate results on the size distribution and likely composition of the dust grains. By varying the mean radius $a_{\mathrm{m}}$, the width of the distribution $\sigma_{\mathrm{m}}$ and the optical constants (i.e. the complex refractive index $m=n-i k$ ), the best fit to the data in Fig. 5 is obtained for an ensemble of particles composed of silicates and carbonaceous material (for instance, graphite), with mean radius $a_{\mathrm{m}} \sim 1.05 \mu \mathrm{m}$ and $\sigma_{\mathrm{m}} \approx 1.3$. The pure silicate models do not reproduce the steady increase of the dust color with wavelength, whereas the pure graphite models produce colors that are too red. The best compromise is obtained for a mixture of both materials.

On the other hand, more complex Mie theory models developed by Lisse (1992) for spherical porous particles of different composition and with a number distribution by mass of $\mathrm{d} n / \mathrm{d} \log m \sim m^{\alpha}$, with $\alpha=-0.56$ can also provide us with color vs. wavelength between 0.5 and $5 \mu \mathrm{m}$ (see Fig. 3 in Berriman et al. 1994). Comparison of the color retrieved from our observations (i.e. flux at 1.65 and $2.205 \mu \mathrm{m}$ relative to that at $1.26 \mu \mathrm{m})$, compared to the model results by Lisse (1992) points to a mixture of porous grains made of silicates and amorphous carbonaceous material as a likely dust composition in the coma of C/1999 T1. As Berriman et al. (1994) points out, the Mie theory calculations do not reproduce the observations in detail, however they help to constraint the size range and composition material of the dust grains responsible for the scattered light in the visible and near-IR.

\section{Conclusions}

The variations of the dust emission in comet C/1999 T1 during this short range of heliocentric distances (i.e. decrease at increasing $r_{\mathrm{h}}$ ) could be the effect of localised and sporadic variation in the dust production and not a real trend of cometary activity as a function of $r_{\mathrm{h}}$. No non-spherical coma structures are present from Jan. 26 to Feb. 04, 2001, and the behaviour of the dust coma can be described by long-lived grains (lifetime $\sim 2-3$ days) expanding radially outwards produced in a steadystate form from the nucleus. This gives rise to brightness profiles following the $\operatorname{law} \log B \sim \log \rho^{-1}$ at $\rho \leq 40000 \mathrm{~km}$. Simple or more complex Mie-theory models point to similar characteristics of the dust coma. Particles composed of a mixture of silicates and carbonaceous material (graphite, for instance), and with a mean radius of $a_{\mathrm{m}} \sim 1.0 \mu \mathrm{m}$, might be responsible for the scattered light in the optical and near-IR range.

Acknowledgements. TIRGO is operated by the Centro per l'Astronomia Infrarossa e lo Studio del Mezzo Interstellare (CAISMIC.N.R.) with the assistance of the Osservatorio Astrofisico di Arcetri and the Dipartimento di Astronomia e Scienza dello Spazio of the Universita' di Firenze.

This research has been partially supported by the Spanish Ministerio de Ciencia y Tecnología under contract PNE-002/2000-C. Based on observations made with the Italian Telescopio Nazionale Galileo (TNG) operated on the island of La Palma by the Centro Galileo Galilei of the INAF (Istituto Nazionale di Astrofisica) at the Spanish Observatorio del Roque de los Muchachos of the Instituto de Astrofisica de Canarias. L.-M.L. thanks F. Moreno for fruitful conversations on dust dynamics.

\section{References}

A'Hearn, M. F., Schleicher, D. G., Feldmann, P. D., Millis, R. L., \& Thompson, D. T. 1984, AJ, 89, 579

Berriman, G. B., Boggess, N. W., Hausser, M. G., et al. 1994, ApJ, 431, L66

Boehnhardt, H., \& Birkle, K. 1994, A\&A, 107, 101

Burns, J. A., Lamy, P. L., \& Soter, S. 1979, Icarus, 40, 1

Campins, H., Rieke, M. J., \& Rieke, G. H. 1989, Icarus, 78, 54

Fulle, M., Mikuz, H., Nonino, M., \& Bosio, S. 1998, Icarus, 134, 235

Gehrz, R. D., \& Ney, E. P. 1992, Icarus, 100, 162

Hanner, M. S., \& Tokunaga, A. T. 1991, in Comets in the post-Halley era, 1,67

Hartmann, W. K., \& Cruikshank, D. P. 1984, Icarus, 57, 55

Jewitt, D. 1991, in Comets in the post-Halley era, 1, 19

Jewitt, D., \& Meech, K. J. 1986, ApJ, 310, 937

Lisse, C. M. 1992, Ph.D. Thesis, Univ. Maryland

Lynch, D. K., Russell, R. W., \& Sitko, M. L. 2002, Icarus, 159, 234

Moreno, F., Lara, L. M., \& Molina, A. 2002, Proc. to the ACM 2002 Conf., ESA-SP, in press

Moreno, F., Lara, L. M., López-Moreno, et al. 2003, A\&A, 399, 789

Soderbloom, L. A., et al. 2002, Science, 296, 1087

Tokunaga, A. T., Goldfisch, W. F., Griep, D. M., Kaminski, C. D., \& Hanner, M. S. 1988, AJ, 96, 1971

Tozzi, G. P., \& Licandro, J. 2002, Icarus, 157, 187 